\title{
Développement des compétences transversales et surtout de la compétence numérique des apprenants dans un projet de télecollaboration entre deux universités du pourtour méditerranéen
}

\author{
Martin, Justine \\ Universidad de Castilla-La Mancha, Justine.Martin@uclm.es
}

\begin{abstract}
Resumen
La telecolaboración (TC) es definida como una técnica que implica grupos de estudiantes en proyectos virtuales con pares de clases de lugares geográficamente alejados. Estos proyectos sirven, por un lado, para mejorar la competencia comunicativa en lengua extranjera y, por otra parte, para el desarrollo de otras competencias transversales. Este trabajo se inscribe en el marco de un proyecto de telecolaboración con intercambios en línea poniendo en relación aprendices tutores de FLE con estudiantes de FLE de nivel B1. La interacción entre los estudiantes de nivel B1 se realiza alrededor de tareas creadas y animadas por los tutores. Nuestro estudio se interroga sobre las competencias transversales desarrolladas, y en particular la competencia digital adquirida, por los estudiantes durante la realización de esas tareas. El proyecto RealBergame (RB) implica estudiantes de universidades de dos países del contorno mediterráneo : la Universitá Delgi Studi di Bergamo (UNIBG) y la Universidad de Castilla-La Mancha (UCLM). En el marco de este proyecto, los tutores han tutorizado a los estudiantes durante seis a ocho semanas. Estas tutorías consistían en animar, a distancia, tareas para estos últimos. Los tutores los guiaban durante la realización de las tareas colaborativas y les brindaban una retroalimentación de sus actividades. Esta ayuda podía ser de orden pedagógico, disciplinaria o técnica. Al estar la comunicación mediatizada por ordenador (CMO), la competencia digital o técnica es una de las competencias esenciales para participar en estos tipos de proyectos. Así pues, hemos analizado los efectos que tienen estos tipos de proyectos en la adquisición de las competencias transversales de los estudiantes, pero sobre todo la competencia digital.
\end{abstract}

Palabras clave : cultura digital ; telecolaboración ; aprendizaje ; competencias transversales.

\section{Résumé}

La télécollaboration (TC) est définie comme une technique qui implique des groupes d'étudiants dans des projets virtuels avec des pairs de classe, de lieux géographiquement distants. Ces projets servent, d'un côté à améliorer la compétence communicative des étudiants, et d'un autre côté à développer d'autres compétences transversales. Cette proposition de communication s'inscrit dans le cadre d'un projet de télécollaboration présentant des échanges en ligne mettant en relation des apprentis tuteurs de FLE avec des apprenants de FLE niveau B1. L'interaction entre ces apprenants de niveau B1 se réalise autour de tâches créées et animées par les tuteurs. Notre étude s'interroge sur les compétences transversales développées, et particulièrement la compétence numérique acquise, par les apprenants lors de la réalisation de ces tâches. Le projet RealBergame (RB) implique des apprenants d'universités de deux pays du pourtour méditerranéen : l'Universitá Delgi Studi di Bergamo (UNIBG) et l'Universidad de CastillaLa Mancha (UCLM). Dans le cadre de ce projet, les tuteurs ont tutoré les apprenants durant six à huit semaines. Ce tutorat consistait à animer, à distance, des tâches pour les apprenants. Ceux-ci leur apportaient une aide pendant la réalisation des tâches collaboratives et leur fournissaient une rétroaction sur leurs activités. Cette aide pouvait être d'ordre pédagogique, disciplinaire ou même technique. Pour y participer, la communication étant médiatisée par ordinateur (CMO), la compétence numérique ou technique de l'apprenant est l'une des compétences essentielles de ce genre de projet. Nous nous sommes donc interrogés sur les effets qu'a ce genre de projet sur les compétences transversales des apprenants mais surtout la compétence numérique. 
Développement des compétences transversales et surtout de la compétence numérique des apprenants dans un projet de télecollaboration entre deux universités du pourtour méditerranéen.

Mots-clés : culture numérique ; télécollaboration ; apprentissage ; compétences transversales.

\begin{abstract}
Telecollaboration (TC) can be defined as a technique involving different groups of students in virtual projects in two types of places geographically far apart. These projects serve, on the one hand, to improve the communicative competence in a foreign language and, on the other, to develop other transversal competences. This study is framed within a telecollaboration project with online exchanges, thus relating FLE learning tutors with FLE students having a B1 level. The interaction among students with a B1 level is accomplished through tasks created and animated by their tutors. Our study questions about the transversal competences that are developed, particularly, the digital competence acquired by students while carrying out those tasks. The project RealBergame (RB) involves students from two universities in different countries in the Mediterranean surroundings: Universitá Delgi Studi di Bergamo (UNIBG) and University of Castilla-La Mancha (UCLM). Within this project, tutors have supervised those students for 6-8 weeks. These supervised sessions consisted of encouraging, in the distance, tasks for students to be done. The tutors guided them during the process of collaborative tasks and provided them with feedback about the activities done. This help could be pedagogical, disciplinary or technical. To take place, as communication was done through computers (CMO), the digital or technical competence is one of the fundamental competences in these types of projects. Therefore, we have analysed the effects these types of projects have on the acquisition of transversal competences of the students but, above all, the digital competence.
\end{abstract}

Keywords : digital culture ; telecollaboration ; learning ; transversal competences.

\title{
Introduction
}

Le développement de certains outils numériques tels que les wikis, les blogs, les vidéo-bulles ou les forums, ont permis une remise en question de nos pratiques d'enseignement des langues étrangères. En ce qui concerne l'apprentissage, l'évolution de ces technologies avance considérablement chaque année de sorte que le développement des compétences transversales de l'apprenant soit devenu nécessaire. L'éducation en générale et les enseignants en particuliers doivent donc veiller à ce que ces compétences soient ancrées au sein d'un ensemble structuré de connaissances afin que les apprenants les acquièrent. Dans un projet de collaboration à distance (ou télécollaboration) en langue française où deux groupes d'apprenants de pays différents, mais au niveau en langue française équivalent, se mettent en contact et collaborent à travers des tâches, nous acceptons la prémisse que pour communiquer efficacement, cette communication doit passer par des canaux numériques ce qui fait que la compétence numérique sera obligatoirement une des compétences transversales développées.

La présente étude s'inscrit dans le cadre d'un projet de télécollaboration en français langue étrangère, et donc vernaculaire, et a pour objectif l'analyse de l'acquisition des compétences transversales de la part des apprenants. Les échanges se réalisent autour d'activités et de tâches numériques suivies et guidées par un-e tuteur-trice. En partant d'une proposition didactique et des retours des apprenants nous souhaitons donc montrer comment la participation dans un projet de ce type permet le développement de certaines de ces compétences transversales.

\section{Contexte}

Les apprenants de première année de l'UCLM (Espagne) et ceux de deuxième année de l'UNIBG (Italie) ont réalisé des tâches dirigées par des tuteurs et tutrices (étudiants de $4^{\text {ème }}$ année de la matière Enseignement du FLE de l'UCLM), à distance. La répartition des apprenants s'est faite en groupes de 4 apprenants par tuteur-tutrice. La communication entre les apprenants et les tuteurs et tutrices a été exclusivement médiatisée par ordinateur à travers la plateforme Moodle ${ }^{1}$ via forum et vidéo-bulles. Que ce soit pour la réalisation du projet ou pour l'organisation et la répartition des tâches, toute

\footnotetext{
${ }^{1}$ www.realbergamemoodle.com.
} 
communication devait être médiatisée par ces moyens. Le projet final pouvait être présenté sous différents formats : blog, vidéo, magazine en ligne, podcast, etc. La seule exigence concernait les outils de communication. Nous avions donc un total de 11 groupes (certains groupes ayant un effectif plus élevé d’apprenants avaient deux tuteurs ou tutrices).

Pour terminer et en nous appuyant sur un inventaire d’objectifs possibles dans un projet de TC, dressé par (Helm, 2013), nous pouvons dire que les principaux objectifs de notre projet sont les suivants :

- Favoriser une communication authentique et les échanges linguistiques dans un contexte universitaire d'apprentissage d'une langue étrangère, ici le FLE ;

- Développement de la compétence TIC : utilisation d’outils numériques, échanges synchrones et asynchrones (vidéosbulles, forum, mail, chat, etc.), tutorat en ligne, appropriation du dispositif, etc. ;

- Développement de la compétence de production et de la compétence interculturelle chez les apprenants italiens et espagnols ;

- Développement de la compétence en autonomie et de la prise de responsabilité ;

- Développement de la capacité à travailler en groupe et à s’y intégrer.

\section{Fondements théoriques}

Le courant socioconstructiviste perçoit l'apprentissage comme un processus émergeant des interactions sociales (Vigotski, 1985) et considère ces dernières comme étant la condition indispensable. Ces théories défendent l’idée que pour une meilleure intégration des savoirs, il faut solliciter les capacités cognitives, sociales et affectives des apprenants. Les compétences transversales permettent ainsi, en parallèle aux compétences disciplinaires, la mobilisation et l'usage de ressources beaucoup plus diverses, dérivant de plusieurs disciplines. Le processus d'apprentissage-acquisition des langues (pour ce qui nous concerne) ne doit donc pas se réduire seulement à un apprentissage ou acquisition d'une langue au sens stricte, mais doit aussi tenir compte du développement de certaines compétences et stratégies. En effet, selon le CECRL (2001), l'apprenant doit « développer des compétences et des stratégies (pour autant qu’ils ne l'aient pas fait auparavant) et exécuter les tâches, les activités et les opérations nécessaires pour participer efficacement à des actes de communication » (Conseil de l'Europe, 2001 : 110). De plus, il insiste sur le fait que l'apprentissage autonome puisse être encouragé si «l'on considère “qu’apprendre à apprendre” fait partie intégrante de l'apprentissage langagier, de telle sorte que les apprenants deviennent de plus en plus conscients de leur manière d'apprendre, des choix qui leur sont offerts et de ceux qui leur conviennent le mieux » (Conseil de l'Europe, 2001, p. 110). Ainsi, l'enseignant devrait mettre en place des scénarios pédagogiques permettant aux apprenants d'apprendre à anticiper de façon proactive, c'est-à-dire en prenant des initiatives, et en planifiant et exécutant leur propre apprentissage.

La commission européenne a donc publié, trois ans plus tard, un cadre européen de référence où elle propose une série de huit compétences clés transversales et essentielles à l'inclusion sociale et au développement de la citoyenneté active. Ces compétences clés pour l'éducation et la formation tout au long de la vie constituent un ensemble de connaissances, d’aptitudes et d'attitudes nécessaires à la vie professionnelle. Selon Tardif \& Dubois :

Les compétences transversales délimitent et ciblent des savoir-agir que tout être humain devrait développer en milieu scolaire afin d'être en mesure de comprendre la complexité des situations de la vie courante et de mettre en œuvre des actions judicieuses (Tardif \& Dubois, 2013 : 29).

Le cadre définit ainsi ces huit compétences clés et décrit cet ensemble de manière assez approfondie :

- La communication dans la langue maternelle : faculté d'exprimer et d'interpréter des concepts, pensées, sentiments, faits et opinions oralement et par écrit (écouter, parler, lire et écrire), et d'avoir des interactions linguistiques appropriées et créatives dans toutes les situations de la vie sociale et culturelle ;

- La communication en langues étrangères: elle implique la médiation et la compréhension des autres cultures, son degré de maîtrise dépendant de plusieurs facteurs et des capacités d’écouter, de parler, de lire et d’écrire ; 
Développement des compétences transversales et surtout de la compétence numérique des apprenants dans un projet de télecollaboration entre deux universités du pourtour méditerranéen.

- La compétence mathématique et les compétences de base en sciences et technologies : il s'agit de l'aptitude à développer et appliquer un raisonnement mathématique afin de résoudre divers problèmes de la vie quotidienne. Les compétences de base en sciences et technologies renvoient à la maîtrise, à l'emploi et à l'application des connaissances et méthodologies qui servent à expliquer le monde de la nature supposant une compréhension des changements liés à l’activité humaine et à la responsabilité de tout individu en tant que citoyen :

- La compétence numérique qui implique l'usage sûr et critique des technologies de la société de l’information (TSI) et, donc, la maîtrise des technologies de l'information et de communication (TIC): l'utilisation de l'ordinateur pour obtenir, évaluer, stocker, produire, présenter et échanger des informations, et pour communiquer et participer via l’internet à des réseaux de collaboration ;

- Apprendre à apprendre : elle est liée à l'apprentissage, à la capacité à entreprendre et organiser soi-même un apprentissage à titre individuel ou en groupe, selon ses propres besoins, à avoir conscience des méthodes et des offres ;

- Les compétences sociales et civiques. La compétence sociale renvoie aux compétences personnelles, interpersonnelles et interculturelles ainsi qu'à toutes les formes de comportement d'un individu pour participer de manière efficace et constructive à la vie sociale et professionnelle. Elle correspond au bien-être personnel et collectif;

- L'esprit d'initiative et d'entreprise : il s’agit de la capacité de passer des idées aux actes interpelant ainsi la créativité, l'innovation et la prise de risques, ainsi que la capacité de programmer et de gérer des projets en vue de la réalisation d’objectifs. L’individu est conscient du contexte dans lequel s’inscrit son travail ;

- La sensibilité et l'expression culturelles : il s’agit de la conscience de l'importance de l'expression créatrice d’idées, d'expériences et d'émotions sous diverses formes (musique, arts du spectacle, littérature et arts visuels).

Selon la commission européenne, la réflexion critique, la créativité, l’initiative, la résolution de problèmes, l'évaluation des risques, la prise de décision et la gestion constructive des sentiments en sont les piliers et ces compétences sont toutes interdépendantes les unes des autres.

\section{Méthodologie}

Notre méthodologie de recherche relève ici d'une démarche descriptive et tente de répondre à trois questions :

- Quels sont les ressentis des apprenants envers leurs compétences (numérique et autres) ?

- Quelle est la perception des apprenants envers le déroulement du projet et ce qu’il leur a apporté ?

- Quelles compétences clés sont développées dans un projet de TC ?

Notre corpus est constitué :

- Des synthèses réflexives des apprenants en fin de projet $(\mathrm{N}=42)$

- D’autoévaluations des apprenants $(\mathrm{N}=42)$

- Des évaluations des apprenants de la part des tuteurs-tutrices. ( $\mathrm{N}=42)$

- Journal de Bord des tuteurs et tutrices $(\mathrm{N}=19)$

\section{Résultats}

Nous allons présenter ici les résultats de notre analyse en incorporant des données quantitatives mais aussi qualitatives. Dans un premier temps nous avons repris les résultats obtenus à travers les autoévaluations réalisées en début et fin de projet par les apprenants et ensuite, nous avons repris les commentaires des apprenants dans leur synthèse réflexive. 


\subsection{Auto-évaluations}

Comme nous l'avons dit plus haut, nous avons réalisé une autoévaluation en amont du projet puis à la fin de ce dernier afin d'observer le ressenti des apprenants sur l'amélioration de leurs compétences. À chacune de ces questions nous leur avons demandé d'évaluer leur degré de maîtrise sur une échelle de 0 à 5, 0 étant «pas de connaissances » et 5 étant « je pense maîtriser le sujet » (0 « pas de connaissances », 1 « J’ai des connaissances que je n’ai pas appliquées », 2 «j’ai des connaissances que j’ai appliquées peu de fois », 3 «j’ai mis en pratique à plusieurs reprises », 4 «j'ai de bonnes pratiques » et 5 « Je pense maîtriser le sujet »).

Il faut savoir que la consigne générale du projet n’imposait en aucun cas l'utilisation de tel ou tel outils, seulement en ce qui concerne les outils de communication : forum et vidéo-bulle. Nous présentons donc, avant de commencer, un tableau reflétant les outils utilisés dans chacun des 11 groupes.

Tableau 1. Outils utilisés

\begin{tabular}{lc}
\hline \multicolumn{1}{c}{ Outils } & Nombre de groupe \\
\hline Forum & 11 \\
Chat & 7 \\
Vidéo-bulle (Hangouts) & 11 \\
Gantter (logiciel organisation) & 7 \\
Blog & 9 \\
Magazine en ligne & 1 \\
Podcats (Audacity) & 1 \\
\hline
\end{tabular}

Le blog, le magazine en ligne ${ }^{2}$ et les podcasts étaient les outils utilisés pour la présentation de leur projet. Gantter, quant à lui est un logiciel d'organisation et répartition des tâches au sein des groupes de travail particulièrement utile dans un projet de TC dans la mesure où il permet un partage de données qui se synchronisent automatiquement. Enfin, comme nous le voyons dans ce tableau, les 11 groupes ont utilisé les outils de communication Forum et Vidéo-bulle, et 7 d'entre eux y ont rajouté un outil Chat afin de pouvoir communiquer entre eux à l'écrit de façon synchrone et non pas seulement de façon asynchrone. Il s’agit ici d'un choix ayant été fait par les apprenants même et mis en place par les tuteurs et les tutrices.

Nous avons ensuite demandé aux apprenants d’évaluer leur propre vision de leur degré de compétence à l’utilisation de forums de discussion.

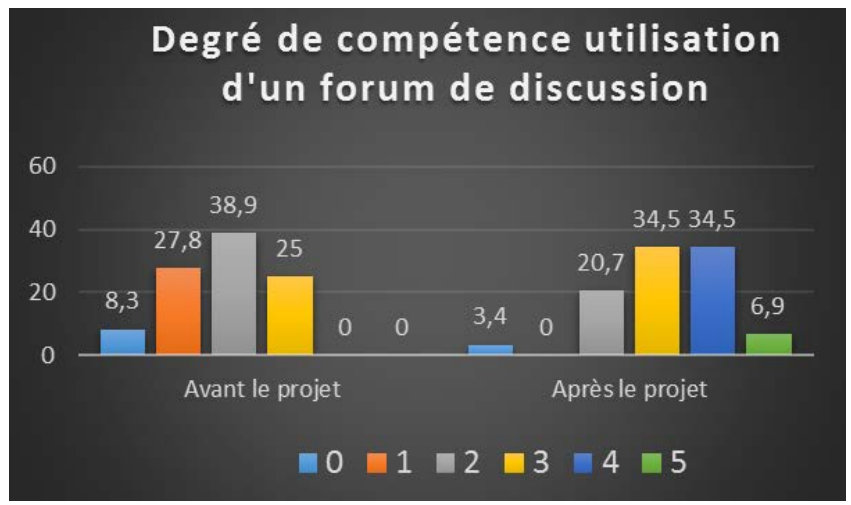

Fig. 1. Degré de compétence à l'utilisation d'un forum

\footnotetext{
${ }^{2}$ Madmagz.
} 
Développement des compétences transversales et surtout de la compétence numérique des apprenants dans un projet de télecollaboration entre deux universités du pourtour méditerranéen.

Selon cette autoévaluation, avant de commencer le projet, aucun des apprenants n'estimaient pouvoir évaluer leur degré de compétence à utiliser un forum (Figure 1) à 4 ou 5. Néanmoins, après l'expérience télécollaborative, on a plus de $40 \%$ des apprenants se considérant dans cette tranche. Il est aussi intéressant de voir que 3,4\% ne voient aucune évolution et considèrent toujours leur compétence à 0 . Si l'on reprend les JdB des tuteurs en ce qui concerne ces apprenants, il s'agit effectivement de personnes ayant participé aux vidéo-bulles, ayant envoyé des mails à leurs tuteurs-tutrices mais n’ayant pas participé aux forums. Néanmoins, considérant les échelles 3, 4 et 5 comme étant ceux qui montrent une utilisation suffisante des outils, il faut souligner que seulement 25\% des apprenants estimaient avant le projet que leur degré de compétence se situait dans cet intervalle contre plus de $75 \%$ à la fin du projet. L’évolution est donc considérable.

Un autre outil utilisé par une grande majorité des apprenants était le blog.

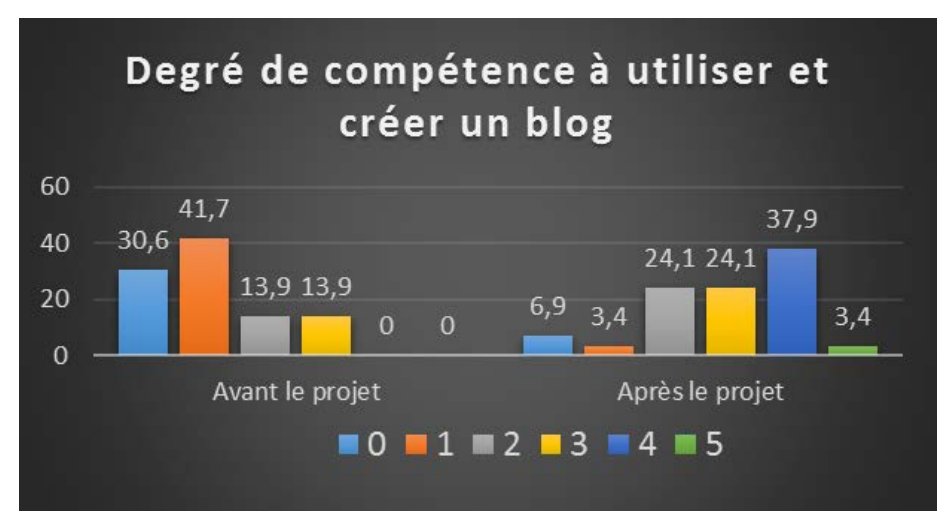

Fig .2. Degré de compétence à utiliser et créer un blog

Lors du projet de TC, deux groupes n’ont pas choisi de réaliser de blog. Cependant 9 des 11 groupes l'ont fait et ont donc dû apprendre à en créer un (Figure 2). Si 30,6\% des apprenants n’avaient jamais créé de blog, à la fin du projet seulement $7 \%$ se trouvent dans ce cas. En ce qui concerne leur degré de maîtrise, soulignons qu’ici, en début de projet, 13,9\% se situaient dans l'intervalle dit de «bonne utilisation » (échelles 3, 4 et 5) contre 65,4\% en fin de projet. On a donc aussi une évolution considérable et significative.

La perception qu’ont les apprenants de leur degré de compétence à travailler en autonomie dans une activité de groupe, à distance (Figure 3) est aussi une donnée intéressante. Effectivement, avant de commencer le projet, 50\% des apprenants n'avaient que très peu d'expérience dans ce domaine, le degré le plus haut ici étant de 3 . Néanmoins, de $50 \%$ on passe, en fin de projet, à 10,3\% ayant désormais 89,6\% des apprenants se situant dans la tranche de « bonne utilisation » (échelle 3 ou 4). Aucun des apprenants n’estiment néanmoins maîtriser ce sujet (échelle 5).

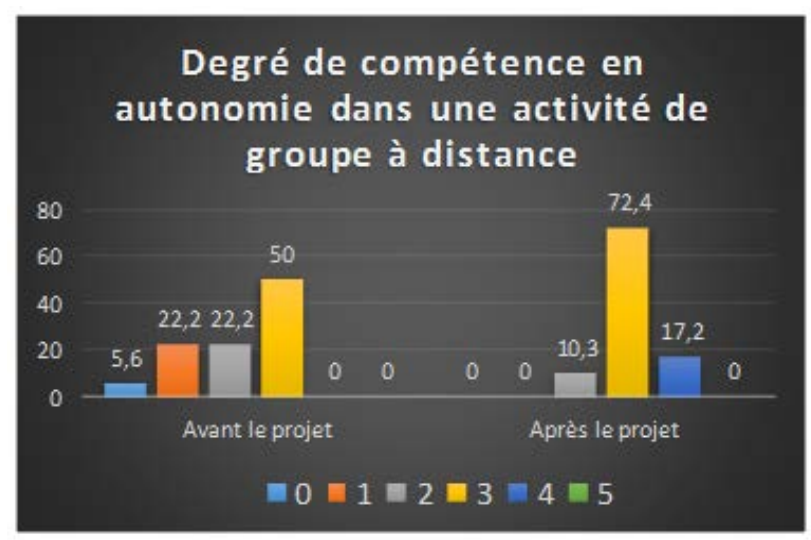

Fig. 3. Degré de compétence à travailler en autonomie dans une activité de groupe à distance 
Globalement, 96,6\% des apprenants estiment ce projet étant assez bon dans le but de favoriser l'amélioration des compétences techniques, linguistiques, à travailler en groupe et en autonomie (Tableau 2), ce dernier étant le seul critère ayant fait l’unanimité puisque la note la plus basse attribuée est un 3/5.

Tableau 2. Note globale pour l'amélioration des compétences dans un projet de TC

\begin{tabular}{|c|c|c|c|c|c|c|c|}
\hline Item & $\begin{array}{c}0 \\
\text { Non } \\
\text { pertinent }\end{array}$ & $\begin{array}{c}1 \\
\text { Mauvais }\end{array}$ & $\begin{array}{c}2 \\
\text { Moyen }\end{array}$ & $\begin{array}{c}3 \\
\text { Assez bien }\end{array}$ & $\begin{array}{c}4 \\
\text { Bien }\end{array}$ & $\begin{array}{c}5 \\
\text { Très bien }\end{array}$ & Moyenne \\
\hline $\begin{array}{c}\text { L’AMÉLIORATION des } \\
\text { compétences } \\
\text { TECHNIQUES }\end{array}$ & $0 \%$ & $0 \%$ & $3,4 \%$ & $34,5 \%$ & $41,4 \%$ & $20,7 \%$ & 3.8 \\
\hline $\begin{array}{c}\text { L’AMÉLIORATION de la } \\
\text { compétence À } \\
\text { TRAVAILLER EN } \\
\text { GROUPE }\end{array}$ & $0 \%$ & $0 \%$ & $3,6 \%$ & $21,4 \%$ & $53,6 \%$ & $21,4 \%$ & 3.9 \\
\hline $\begin{array}{l}\text { L'AMÉLIORATION de la } \\
\text { compétence } \\
\text { LINGUISTIQUE }\end{array}$ & $0 \%$ & $0 \%$ & $3,4 \%$ & $27,6 \%$ & $58,6 \%$ & $10,3 \%$ & 3.8 \\
\hline $\begin{array}{c}\text { L'AMÉLIORATION de la } \\
\text { compétence À } \\
\text { TRAVAILLER EN } \\
\text { AUTONOMIE }\end{array}$ & $0 \%$ & $0 \%$ & $0 \%$ & $32,1 \%$ & $50 \%$ & $17,9 \%$ & 3.9 \\
\hline
\end{tabular}

\subsection{Commentaires des synthèses / bilan réalisés par les apprenants}

Ici nous avons repris certains critères mentionnés par les apprenants dans leur synthèse finale à travers leurs commentaires qui démontrent que le projet aurait eu, selon eux, une influence sur le développement d'une des compétences mentionnées plus haut. Nous avons relevé certains de ces derniers qui montrent leur perception de l'évolution de leurs compétences transversales. Nous avons donc repris des commentaires ${ }^{3}$ se référant à ces dernières. La compétence en langue étrangère est une compétence qui a déjà été nommée plus haut, nous ne reviendrons donc pas dessus.

\section{- La compétence numérique}

La compétence numérique est une des compétences dont l’évolution est la plus évidente pour eux ainsi que pour nous, enseignant et pour les tuteurs et tutrices. La création de blogs est ce qui revient le plus :

Faire un blog c'est aussi une chose positif parce que avant le project, je ne savais pas comme je peux faire un, mais maintenant je le sais. App_esp_1

J’ai aussi apprécié la réalisation du projet car je n’avais jamais créé un blog, donc ça était tout nouveau pour moi ?j! App_esp_

Je pense que ce project ètait très interessant parce que il m’à donné la chance de connaitre des nouveaux amis et d'apprendre a utiliser des nouveaux programmes comme hangout ou wordpress. App_it_1

De plus, avec ce projet j’ai appris de comment créer et utiliser un blog, comment utiliser Sound Cloud, comment faire un hangout, comment utiliser la plateforme de Real Bergame, comment faire un débat et comment créer un forum de débat entre autres. App_esp_3

\footnotetext{
${ }^{3}$ Les écrits sont anonymes. Les paroles des apprenants sont reprises telles quelles sans aucune modification de notre part.
} 
Développement des compétences transversales et surtout de la compétence numérique des apprenants dans un projet de télecollaboration entre deux universités du pourtour méditerranéen.

D’autres restent plus généraux mais mentionnent aussi ce critère dans leur synthèse :

D’autre réussite dans cette expérience est l’apprentissage que j’ai acquis sur la technologie. App_esp_4

\section{- Les compétences sociales et civiques}

Ces compétences sont fondées sur la collaboration, la confiance en soi et l'intégrité. L’apprenant doit ici démontrer qu’il a de l'intérêt pour la communication interculturelle, et doit valoriser la diversité et le respect de l'autre.

Aussi, nous avons pu les connaitre mieux, surtout sa culture. App_esp_5

nous avons amélioré nos compétences sociales et notre forme de travail en groupe, aussi bien que nous avons également élargi notre connaissance du monde et enrichi notre culture. App_esp_7

Et finalement je voudrais ajouter que, grâce à ce projet, je devais parler beaucoup avec ma collègue italienne et elle a été une grande aide pour mon français aussi. Maintenant, nous avons une proposition d'apprendre l'italien et l'espagnol entre nous. App_esp_2

D’abord j’ai acquis un peu plus de sécurité en ce qui concerne la conversation orale parce que grâce aux visioconférences Hangout j’ai mis de côté ma timidité pour essayer d'entrer en contact et parler avec d'autres personnes. App_it_2

Il doit aussi être prêt à vaincre les préjugés et à accepter des compromis ;

La partie positive a été de travailler avec des étudiants d'autres groupes de notre université et d'autres pays. C’est bon parce que nous connaissons d'autres cultures et différentes façons de travailler. App_esp_8

Pendant ce projet, j’ai vraiment amélioré mon français orale et écrit, j’ai connu nouvelles personnes, nouvelles cultures et particularités intéressantes qui caractérisent les différents pays. App_it_3

\section{- Apprendre à apprendre}

Apprendre à apprendre exige que l'apprenant connaisse, ou du moins découvre, ses stratégies d'apprentissage préférées, ses points forts et ses points faibles en ce qui concerne ses aptitudes et qualifications. De plus, il devra être capable de s’organiser afin de mener à bien les tâches que l'on attendra de lui ou elle autrement dit, il devrait être capable d'organiser son propre apprentissage.

Nous avons su organiser correctement les devoirs. App_esp_5

c'est une bonne expérience et un travail très laborieux parce que nous avons pris beaucoup de temps à rechercher l’information et la corriger. App_esp_9

À travers cette compétence, l'apprenant doit aussi être capable de surmonter les obstacles ce qui révèle une attitude positive orientée vers la résolution de problèmes.

C’est vrai qu’il a été un peu difficile à coordonner au début, mais quand on a commencé à travailler, ça a été bien en général. App_esp_10

Nous avons bavardé ensemble sur WhatsApp et corriger les erreurs de l'autre. App_it_4 
J'ai le sentiment d'être plus fort en groupe pour affronter la tâche finale sur un thème articulé comme la Francophonie. App_it_5

Enfin, l'apprenant doit pouvoir démontrer qu'il peut passer du temps à apprendre de façon autonome, mais aussi à travailler en équipe dans le cadre du processus d'apprentissage.

Dans ce travail, j’ai appris comment travailler d’une façon autonomique et plus individuelle. App_esp_11

Je pense que le projet m’a aidé à apprendre à travailler en groupe à distance. App_it_6

Ces sont les bonnes choses, mais c'est vrai qu'il y a un problème dans ce projet, le retard, je crois que si nous avions commencé plus tôt, les résultats auraient pu être mieux. App_esp_12

\section{- L'esprit d'initiative et d'entreprise}

Cette compétence est ici fondamentale dans un tel projet. Les aptitudes correspondantes à cette dernière relèvent de la gestion de projet, c’est-à-dire de la capacité à planifier, à organiser, à gérer des situations de travail collectif, à déléguer, à arriver à des consensus, à la communication. Elles relèvent aussi d'une disposition à prendre des initiatives et impliquent la motivation et détermination à réalisera les objectifs poursuivis.

je préfère être dans un groupe, juste au cas où je sais que je peux compter sur les autres. App_it_7

on devrait être plus responsable avec nos obligations, même si parfois ils peuvent sembler insignifiants. App_esp_13

Tous les membres de mon groupe ont écrit une partie de la revue. Les membres de mon groupe prennent en compte les propositions de tous. App_it_8

Nous sommes arrivés à un consensus même s’il y a eu quelques incompréhensions. App_it_9

Comme dans tous les groupes de travail, il y a toujours des gens qui travaillent plus que d'autres, mais la solution est qu'on doit mettre de l'intérêt et encourager et tous les membres du groupe doivent participer et donner leur opinions.

App_esp_14

j’ai pu améliorer la langue française un peu et, aussi, travailler d’une façon différente mais productive. App_esp_15

Nous prenions en compte nos propositions, nous encouragions... et nous prenions d'accord avec le meilleur choix. App_esp_16

Nous avons discuté des différents points de vue pour choisir le thème du Blog. App_esp_16

Ces commentaires relèvent aussi de la résolution de problèmes étant donné qu'après les conflits, les apprenants ou plus exactement les groupes d’apprenants sont arrivés à un consensus et en sont tout à fait conscient.

\section{- La sensibilité et l'expression culturelle}

Cette compétence s'exprime à travers leurs créations, les différents projets réalisés sous format blog ou podcast ou encore à travers la réalisation artistique d'une revue web.

Six des huit compétences sont donc mentionnées dans leur écrit et se retrouvent à travers leur auto évaluation.

\section{Conclusion}

Les environnements numériques de travail de type Moodle nous offrent des opportunités de développer une méthodologie d'enseignement/apprentissage basées sur le travail collectif à distance. Ces possibilités nous obligent à redessiner nos scénarios pédagogiques et à repenser des situations de communication permettant alors la communication asynchrone. Ces changements favorisent, comme nous l’avons vu l’acquisition de compétences transversales nécessaires au 
Développement des compétences transversales et surtout de la compétence numérique des apprenants dans un projet de télecollaboration entre deux universités du pourtour méditerranéen.

développement cognitif, social et affectif de l'apprenant dans la mesure où elles mettent en jeu des stratégies et des situations difficilement accessibles dans des situations d'enseignement-apprentissage classiques.

Dans ce travail nous voulions faire remarquer que grâce à une scénarisation adaptée, les pratiques collaboratives à distance des apprenants à travers une plateforme LMS non seulement favorisent leur expression et l'interaction mais apparaît aussi comme une opportunité professionnelle d'innovation.

\section{Références bibliographiques}

CONSEIL De L’EuROPE (2001). Cadre européen commun de référence pour les langues : apprendre, enseigner, évaluer. Paris : Didier $<$ https://www.coe.int/t/dg4/linguistic/Source/Framework_FR.pdf $>$ [Consultée le 01 juin 2016].

De Lavergne, Catherine, \& HeÏD, Marie-Caroline (2013). «Former à et par la collaboration numérique: quels enjeux pour l'enseignement universitaire ? » dans Tic\&société, Vol.7, Nº1, p. 116-138. < http://ticetsociete.revues.org/1308> [Consultée le 03 juin 2016]

Demaiziere, Françoise (2007). «Didactique des langues et TIC : les aides à l'apprentissage » dans Alsic, Vol. 10, N¹, p. 5-21. <http://alsic.revues.org/220> [Consultée le 01 juin 2016]

Guitert, Montse, Romeu, Teresa \& Perez-Mateo, María (2007). « Competencias TIC y trabajo en equipo en entornos virtuales » dans Revista de Universidad y Sociedad del Conocimiento, Vol. 4, $\mathrm{N}^{\circ} 1, \quad$ p. $\quad 1-12$.

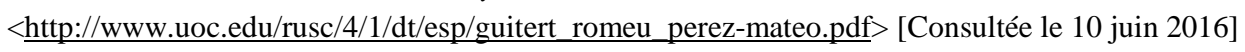

HeLM, Francesca (2013). «A Dialogic Model of Telecollaboration » dans Bellaterra Journal of Teaching \& Learning Language \& Literature, Vol. 19, N 2, p. 28-48. <http://llt.msu.edu/issues/june2015/helm.pdf> [Consultée le 10 juin 2016]

TARDIF, Jacques \& DuBOIs, Bruno (2013). « De la nature des compétences transversale jusqu’à leur évaluation : une course à obstacles, souvent infranchissabes" dans Revue Française de Linguistique Appliquée, Vol. XVIII, No1, p. 29-45. < http://www.cairn.info/revue-francaise-de-linguistique-appliquee-2013-1-page-29.htm> [Consultée le 11 juin 2016]

Vigotski, Lev (1985). Pensée et Langage. Paris : Editions Sociales. 